\title{
Effects of mineralocorticoid receptor blockade on empathy in patients with major depressive disorder
}

\author{
Katja Wingenfeld $^{1} \cdot$ Linn K. Kuehl $^{1} \cdot$ Isabel Dziobek $^{2} \cdot$ Stefan Roepke $^{1}$. \\ Christian Otte $^{1} \cdot$ Kim Hinkelmann $^{1}$
}

Published online: 6 July 2016

(C) Psychonomic Society, Inc. 2016

\begin{abstract}
Background The mineralocorticoid receptor (MR) is highly expressed in the hippocampus and prefrontal cortex and is involved in social cognition. We recently found that pharmacological stimulation of the MR enhances emotional empathy but does not affect cognitive empathy. In the current study, we examined whether blockade of the MR impairs empathy in patients with major depressive disorder (MDD) and healthy individuals.

Methods In a placebo-controlled study, we randomized 28 patients with MDD without psychotropic medication and 43 healthy individuals to either placebo or $300 \mathrm{mg}$ spironolactone, a MR antagonist. Subsequently, all participants underwent two tests of social cognition, the Multifaceted Empathy Test (MET) and the Movie for the Assessment of Social Cognition (MASC), measuring cognitive and emotional facets of empathy.

Results In the MET, we found no significant main effect of treatment or main effect of group for cognitive empathy but a highly significant treatment by group interaction $(\mathrm{p}<0.01)$. Patients had higher cognitive empathy scores compared to controls in the placebo condition but not after spironolactone. Furthermore, in the spironolactone condition reduced cognitive empathy was seen in MDD patients but not in controls.
\end{abstract}

Katja Wingenfeld

katja.wingenfeld@charite.de

1 Department of Psychiatry and Psychotherapy, Charité University Medical Center, Campus Benjamin Franklin, Hindenburgdamm 30, 12203 Berlin, Germany

2 Berlin School of Mind and Brain, Humboldt-University Berlin, Berlin, Germany
Emotional empathy was not affected by MR blockade. In the MASC, no effect of spironolactone could be revealed.

Conclusion Depressed patients appear to exhibit greater cognitive empathy compared to healthy individuals. Blockade of MR reduced cognitive empathy in MDD patients to the level of healthy individuals. Future studies should further clarify the impact of MR functioning on different domains of social cognition in psychiatric patients.

Keywords Mineralocorticoid receptor · Cortisol $\cdot$ Social cognition $\cdot$ Emotional empathy $\cdot$ Cognitive empathy $\cdot$ Major depressive disorder

\section{Introduction}

Major depressive disorder (MDD) is characterized by alterations of the hypothalamus pituitary adrenal (HPA) axis, e.g., higher cortisol release and reduced feedback sensitivity (Parker et al., 2003). Furthermore, patients with MDD often exhibit cognitive deficits (Chamberlain \& Sahakian, 2006; Porter et al., 2007). Interestingly, there is some evidence that higher cortisol levels are associated with impaired memory and executive function (Hinkelmann et al., 2009; Schlosser et al., 2011). Glucocorticoids (GCs) exert their effects by binding to two receptors, the mineralocorticoid receptor (MR) and the glucocorticoid receptor (GR). MRs and GRs differ in their affinity and distribution within the brain: while MRs are mainly located in the hippocampus and the prefrontal cortex, GRs are expressed throughout the brain (de Kloet et al., 2005; Joels et al., 2008; Lupien \& Lepage, 2001; Roozendaal et al., 2010). Furthermore, the two receptor types differ in their binding properties with (intracellular) MRs having a six- to tenfold higher affinity for cortisol (de Kloet et al., 2005). Thus, under 
basal conditions MRs are substantially occupied, while GRs only become heavily occupied under stress.

While most of the effects of GCs on cognition have been attributed to GRs, more recent studies emphasize the importance of MRs in the context of cognitive performance (de Kloet, 2010; Harris et al., 2012; Joels et al., 2008). Several studies showed that blocking the MR with spironolactone leads to impaired cognitive function in humans (Cornelisse et al., 2011; Otte et al., 2007; Rimmele et al., 2013). Interestingly, these impairing effects of MR blockade were most pronounced for emotional memory (Rimmele et al., 2013).

In a series of studies, we investigated the effects of pharmacological stimulation of GR and/or MR in patients with MDD and in healthy controls. We found that stimulation of both receptors via hydrocortisone administration did not affect cognitive performance, i.e., word list learning, autobiographical memory retrieval, working memory, and response inhibition, in MDD patients in contrast to healthy controls (Schlosser et al., 2010; Schlosser et al., 2013; Terfehr et al., 2011a, b). These results are in line with the hypothesis of reduced GR sensitivity in MDD (Holsboer, 2000; Webster et al., 2002). Furthermore, (selective) MR stimulation with fludrocortisone led to improved performance of verbal memory and executive function compared to placebo in healthy participants as well as in patients with MDD (Otte et al., 2015).

Stress and/or glucocorticoids influence a wide range of cognitive and emotional functions such as memory performance, fear-motivated behavior, and stress-associated emotional reactions (de Kloet, 2013). Furthermore, there is some evidence that psychosocial stress also impacts social cognition, e.g., empathy (Tomova et al., 2014; Smeets et al., 2009; von Dawans et al., 2012). Empathy consists of at least two components: The first is a cognitive component, which captures the capacity to infer others' mental states and is also referred to as perspective taking, mentalizing, or theory of mind (Zaki \& Ochsner, 2012). The second is an affective component, i.e., an emotional response to another person's emotional state (Blair, 2008; Roepke et al., 2012). Two examples for valid tasks in the field of measuring empathy are the "Movie for the Assessment of Social Cognition" (MASC) (Dziobek et al., 2006) and the "Multifaceted Empathy Test" (MET) (Dziobek et al., 2008). The MASC is a video-based task and participants are required to infer emotions, thoughts, and intentions of characters engaged in social interactions. The MET consists of emotional valent pictures and measures both components of empathy, i.e., emotional and cognitive empathy.

The MET has previously been used to investigate the effects of psychosocial stress on empathy in healthy participants (Wolf et al., 2015). Stress exposure, compared to placebo, led to higher emotional empathy, while cognitive empathy was not affected. In addition, the MET has been used in numerous studies aiming at characterizing empathic functions in psychiatric disorders. Of note, diverging patterns of cognitive and emotional empathy deficits have helped distinguishing diagnoses with known involvement of callous-unemotional traits and autism spectrum disorder symptomatology, respectively (Dziobek et al., 2008; Preller et al., 2014). Furthermore, personality traits and pathology have been associated with cognitive and emotional empathy dysfunction as measured with the MET. For example, while individuals with narcissistic personality disorder have been identified as involving low emotional empathy, borderline personality disorder seems to present with both emotional and cognitive empathy reductions (Dziobek et al., 2011; Ritter et al., 2011).

Studies that examined social cognition in MDD have revealed equivocal results (Schreiter et al., 2013). Of note, there are some studies, which investigated empathy using the MASC and the MET. While one study did not find any deficits in depressed patients in the MASC (Wilbertz et al., 2010), others reported impaired cognitive empathy in the MASC in MDD (Wolkenstein et al., 2011). Interestingly, in the same sample performance in the 'Reading the Mind in the Eyes test' was better in MDD patients compared to controls. Using the MET, no alterations in MDD patients have been found (Thoma et al., 2011). However, to the best of our knowledge the impact of stress or stress hormones on empathy has not been investigated in MDD yet.

In one of our own studies using these tasks we demonstrated that MR stimulation enhanced emotional empathy in the MET but had no effect on cognitive empathy in the MET and in the MASC in healthy women and women with borderline personality disorder (Wingenfeld et al., 2014). Another study also used the MASC to investigate the association between the cortisol response to a psychosocial stressor and cognitive empathy (Smeets et al., 2009). In women, elevated cognitive empathy was found for those who responded with low cortisol to psychosocial stress exposure. Furthermore, in women there was a negative association between cortisol reactivity and MASC scores (Smeets et al., 2009). In men, an opposite pattern was seen, with high cortisol responders showing greater MASC scores and a negative correlation between stress hormones and empathy. Thus, this study suggests sex-specific effects of glucocorticoids on social cognition. However, stress-induced endogenous cortisol release targets both GR and MR and, thus, the related effects are not distinguishable.

In the current study we aimed to further investigate the role of the MR in social cognition, i.e., empathy. We decided to use the MET and the MASC again, as in our previous study on MR function and empathy in healthy subjects and patients with borderline personality disorder. In that study, we found that MR stimulation increased emotional empathy (Wingenfeld et al., 2014). Thus, we aimed to investigate whether MR blockade has the opposite effects. Because other groups have shown 
that stress influences performance in the MASC (Smeets et al., 2009), we decided to also test the effects of MR blockade in this task, especially as cognitive empathy in the MASC was found to be impaired in MDD (Wolkenstein et al., 2011).

Since stimulation of the MR had positive effects on emotional empathy in our previous study (Wingenfeld et al., 2014), we hypothesized that blocking the MR would lead to impaired social cognition. Indeed, it has been consistently shown that blocking the MR leads to impaired cognitive function in humans (Cornelisse et al., 2011; Otte et al., 2007; Rimmele et al., 2013). As studies on empathic abilities in MDD revealed inconclusive results (Schreiter et al., 2013), we further aimed to investigate whether patients with MDD show altered cognitive and emotional empathy when using ecological tasks like the MET and the MASC, and whether empathy in depressed patients might be modulated by MR blockade.

\section{Materials and methods}

\section{Participants}

Twenty-eight patients with MDD and 43 healthy participants were recruited and completed the study. Patients included inand outpatients recruited from a specialized depression clinic at the Department of Psychiatry, Charité Campus Benjamin Franklin, Berlin, Germany. The control group was recruited by public postings, e.g., via our research website and matched for age, sex, and years of education.

Inclusion criteria for patients with MDD were: (1) a diagnosis of major depressive disorder, single or recurrent according to DSM-IV criteria, assessed by the MINI-International Neuropsychiatric Interview (MINI; Sheehan et al., 1998) German version (Ackenheil et al., 1999) and (2) a minimum baseline score of 18 points on the Hamilton Rating Scale for Depression, 17-item version (HDRS-17; Williams, 1988). Severity of depressive symptoms was assessed with the Beck Depression Inventory (BDI; Beck \& Steer, 1994).

All participants were free of psychotropic medication. Participants were excluded if they had any of the following medical conditions: CNS diseases or severe somatic diseases, metabolic or endocrine diseases, autoimmune diseases, current infections, or pregnancy. Further exclusion criteria were schizophrenia, schizoaffective disorder, bipolar disorder, depressive disorder with psychotic features, eating disorder, alcohol or drug abuse, and dependence within the last 6 months (all assessed by MINI). In addition, in the control group any psychiatric diagnosis assessed by the MINI lead to exclusion.

Written informed consent was obtained from all participants. Healthy participants were recruited by local advertisement and received financial remuneration $(80 €)$. The study was approved by the Medical Councils' Ethics Committee of Hamburg and Berlin.

\section{Procedure}

A placebo-controlled study was performed and participants were randomized to either $300 \mathrm{mg}$ spironolactone (Aldactone, Roche Austria, Vienna, Austria) orally or to placebo. Drugs were administered at 14:00 $\mathrm{h}$ and participants underwent cognitive testing between $15.45 \mathrm{~h}$ and $16.45 \mathrm{~h}$. We did not use a within-subject design because no parallel versions of the MET and MASC were available. The participants were tested in a quiet room and were allowed to drink some water.

Spironolactone is a synthetic mineralocorticoid receptor antagonist with additional antagonistic effects on the androgen receptor and some agonistic effects on the progesterone receptor. Peak serum concentrations of the drug are reached within $1-3 \mathrm{~h}$ after oral administration; protein binding is greater than $91 \%$ and the elimination half-life is $1.3-1.4 \mathrm{~h}$.

\section{Social cognition tasks}

\section{Multifaceted Empathy Test (MET)}

To assess cognitive and emotional empathy, the Multifaceted Empathy Test (MET) was used (Dziobek et al., 2008) in a modified version (Dziobek et al., 2011; Ritter et al., 2011). The MET is a PC-assisted test consisting of 30 pictures of people in emotionally charged situations. To assess cognitive empathy, participants were required to infer the mental state of the subject in the picture and indicate the correct emotion from a list of four. To assess emotional empathy, participants were asked to rate the degree of empathic concern they felt for the person in the picture (on a Likert scale; $0=$ not at all, $9=$ very much). Pictures were presented in six blocks of ten picture stimuli. Each block is presented twice, once to be rated for cognitive and once to be rated for emotional empathy. Blocks of emotional and cognitive empathy occurred in alternating order, but the same block is not presented consecutively. The order of the six blocks is thus pseudo-randomized.

\section{Movie for the Assessment of Social Cognition (MASC)}

Additionally, we administered the Movie for the Assessment of Social Cognition (MASC), a sensitive video-based test for the evaluation of cognitive empathy (Dziobek et al., 2006; Preissler et al., 2010; Ritter et al., 2011). A 15-min movie about four characters spending an evening together is shown and questions relating to the actors' mental states are answered. The movie stops at 45 times, then questions about the actors' feelings/emotions (score range $0-15$ ), thoughts (score range 0-4), and intentions (score range 0-14) are asked. 
Participants are required to choose the correct answer out of four possible ones.

\section{Statistical analysis}

Statistical analyses were performed using SPSS Version 19.0. Demographic data were analyzed using Pearson's $\mathrm{Chi}^{2}$ test for categorical data and ANOVA for continuous data. Effects of spironolactone on social cognition were analyzed using analysis of variance (ANOVA), with the main factors treatment (spironolactone vs. placebo) and group (MDD vs. controls). Partial $\eta^{2}\left(\mathrm{p} \eta^{2}\right)$ is given as effect size $(<.04$ small effect, .04-.13 medium effect, $\geq .14$ small effect). In addition, Pearson's correlation analyses between BDI scores and empathy scores were performed separately for the placebo and the spironolactone conditions.

\section{Results}

\section{Demographic and clinical data}

In MDD patients the following current co-morbid axis I disorders were reported: dysthymia $n=2$, panic disorder $n=1$, agoraphobia $\mathrm{n}=1$, PTSD $\mathrm{n}=1$.

MDD patients and healthy controls did not differ with regard to age, sex, years of education, body mass index, and intake of oral contraceptives. There were more smokers in the patient group (sample characteristics are presented in Table 1). As expected, MDD patients had higher BDI scores. The HDRS was only applied in patients but not in healthy controls. There were no differences concerning demographic data between participants receiving placebo and spironolactone, neither in the control group nor in the MDD group.

\section{Effects of spironolactone versus placebo on cognitive and emotional empathy}

In this placebo-controlled study, 13 MDD patients and 24 healthy controls received placebo, while 15 MDD patients and 19 healthy participants received spironolactone. There were missing data for one MDD patient and two controls in the MASC.

\section{MET}

We performed two ANOVAs analyzing the effect of spironolactone (between subject factor '"treatment") in patients with MDD and healthy controls (between subject factor "group"). Emotional and cognitive empathy score served as dependent variable, respectively.

For cognitive empathy there was no significant main effect of treatment $\left(\mathrm{F}_{\mathrm{df1}, 67}=0.59, \mathrm{p}=0.45, \mathrm{p}^{2}=.009\right)$ or group $\left(\mathrm{F}_{\mathrm{df} 1,67}=0.51 \mathrm{p}=.48, \mathrm{p \eta}^{2}=.008\right)$, but a significant treatment by group interaction $\left(\mathrm{F}_{\mathrm{df} 1,67}=7.60 \mathrm{p}=.008, \mathrm{p \eta}^{2}=.1\right)$. To clarify this interaction, post-hoc ANOVAs were conducted for each group (MDD/controls) and each treatment condition (spironolactone/placebo). Patients had higher cognitive empathy test scores compared to controls in the placebo condition $(p=.01)$ while there were no group differences in the spironolactone condition $(\mathrm{p}=.16)$. Furthermore, a significant difference between placebo and spironolactone condition was seen in patients with MDD patients $(p=.02)$ but not in healthy participants $(p=.14)$. Thus, results indicate that spironolactone seems to reduce cognitive empathy only in MDD (See Fig. 1).

Table 1 Sample characteristics

\begin{tabular}{|c|c|c|c|c|c|}
\hline & \multicolumn{2}{|l|}{ MDD } & \multicolumn{2}{|l|}{ Healthy controls } & \multirow[t]{2}{*}{ Statistics } \\
\hline & Placebo $(\mathrm{N}=13)$ & Spironolactone $(\mathrm{N}=15)$ & Placebo $(\mathrm{N}=24)$ & Spironolactone $(\mathrm{N}=19)$ & \\
\hline Age $($ mean/SD) & $41.6(7.2)$ & $41.9(7.6)$ & $39.6(7.6)$ & $40.9(8.5)$ & n.s. \\
\hline $\begin{array}{l}\text { Years of school } \\
\text { education (mean/SD) }\end{array}$ & $10.8(1.5)$ & $11.8(1.5)$ & $11.3(1.6)$ & $11.2(1.4)$ & n.s. \\
\hline $\begin{array}{l}\text { Body mass index } \\
\mathrm{r}(\mathrm{mean} / \mathrm{SD})\end{array}$ & $25.4(7.5)$ & $28.9(9.8)$ & $26.4(5.7)$ & $26.0(5.2)$ & n.s \\
\hline Sex (female/male) & $6 / 7$ & $8 / 7$ & $13 / 11$ & $10 / 9$ & n.s. \\
\hline Smoker (yes/no) & $8 / 5$ & $9 / 6$ & $7 / 16$ & $8 / 11$ & $\begin{array}{l}\mathrm{p}=.04(\mathrm{MDD} \\
\text { vs. controls })\end{array}$ \\
\hline $\begin{array}{l}\text { Use of oral contraceptives } \\
\text { (yes/no) }\end{array}$ & $0 / 13$ & $1 / 14$ & $3 / 21$ & $2 / 17$ & n.s. \\
\hline $\mathrm{BDI}($ mean/SD) & $35.5(9.5)$ & $32.7(8.9)$ & $2.2(2.5)$ & $2.3(3.0)$ & $\begin{array}{l}\mathrm{p}<.001(\mathrm{MDD} \\
\text { vs. controls) }\end{array}$ \\
\hline HRSD (mean/SD) & $26.1(5.9)$ & $23.3(3.8)$ & & & $\begin{array}{l}\text { n.s. Range: } \\
\quad 18-43\end{array}$ \\
\hline
\end{tabular}

$M D D$ major depressive disorder, $B D I$ Beck Depression Inventory, HRSD Hamilton Rating Scale for Depression 


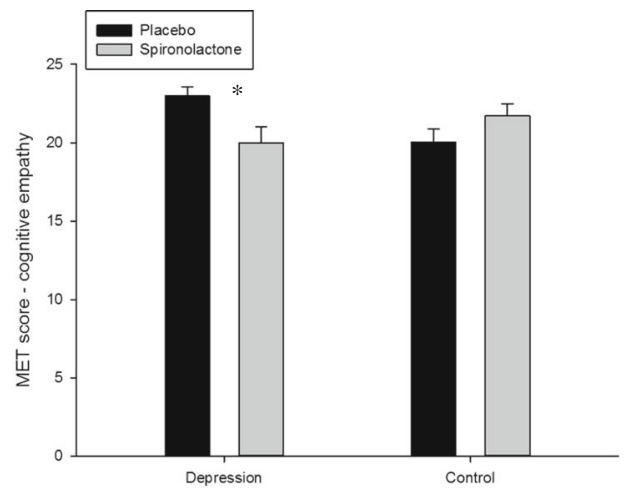

Fig. 1 Cognitive and emotional empathy measured with the Multifaceted Empathy Test (MET) after administration of spironolactone and placebo in patients with major depressive disorder (MDD) and healthy participants. A significant treatment by group interaction effect was found for the cognitive test part $(p=.008)$. Depressed patients performed worse

Regarding emotional empathy no significant main effect of treatment $\left(\mathrm{F}_{\mathrm{dfl}, 67}=0.02, \mathrm{p}=.90, \mathrm{p}^{2} \leq .001\right)$, group $\left(\mathrm{F}_{\mathrm{df1}, 67}=\right.$ $\left.2.61 \mathrm{p}=.11, \mathrm{p \eta}^{2}=.04\right)$, or treatment by group interaction $\left(\mathrm{F}_{\mathrm{df1}, 67}=0.01, \mathrm{p}=.99, \mathrm{p}^{2} \leq .001\right)$ emerged. Thus, MR blockade had no effect on emotional empathy (See Fig. 1).

On a descriptive level, patients showed a lower score on emotional empathy compared to healthy controls. Thus, for explorative purpose, we decided to rerun our analyses taking valence of the stimuli, i.e., positive versus negative into account. We found a significant valence by group interaction $\left(F_{d f 1,67}=20.78 p<.001\right)$, with lower test scores in the MDD patients for positive emotions, but not for negative emotions. Again, there was no main effect of treatment $\left(\mathrm{F}_{\mathrm{df1}, 67}=\right.$ $0.02, \mathrm{p}=.90)$ or group $\left(\mathrm{F}_{\mathrm{df1}, 67}=2.61 \mathrm{p}=.11\right)$ or any further interaction (treatment by group: $F_{d f 1,67}=0.01, p=.99$, valence by drug: $F_{d f 1,67}=2.77 \mathrm{p}=.10$ ) (see Fig. 2).

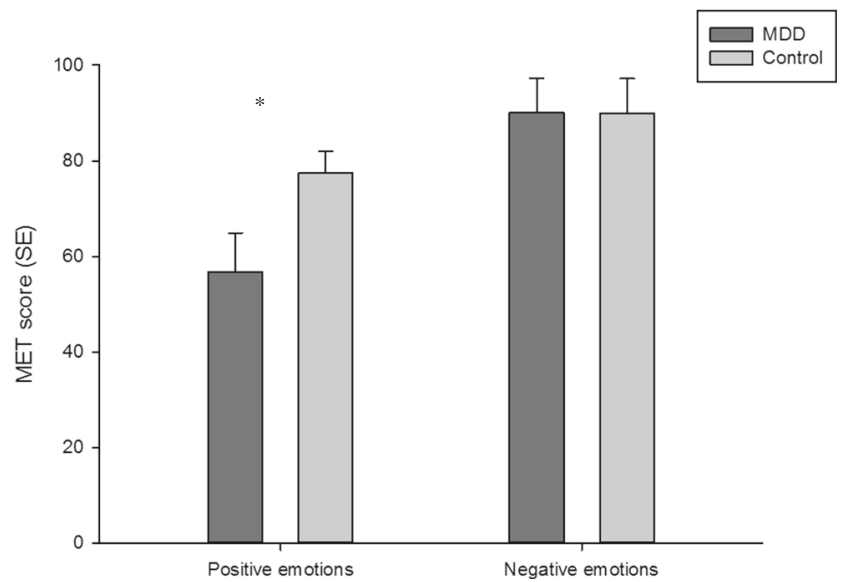

Fig. 2 Emotional empathy measured with the Multifaceted Empathy Test (MET). Test scores (mean and standard error of mean) are given separately for negative and positive stimuli. There was a significant valence by group interaction $(\mathrm{p}<.001)$, with lower test scores in the major depressive disorder (MDD) patients for positive emotions, but not for negative emotions

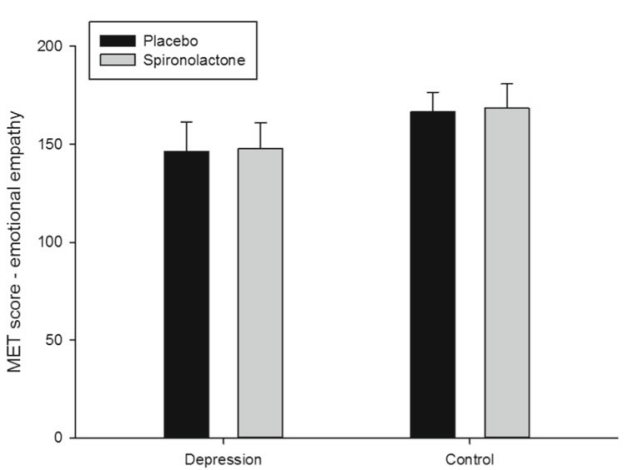

after spironolactone administration compared to placebo $(\mathrm{p}=.02)$. Patients had higher tests scores compared to controls in the placebo condition $(\mathrm{p}=.01)$. Mean and standard error of mean are given for cognitive and emotional empathy score

For explorative purposes, we repeated our analyses including sex as additional factor. There was no effect of sex and the results remained unchanged (emotional empathy: $\mathrm{F}_{\mathrm{df1}, 71}=$ $0.09, \mathrm{p}=.86$, cognitive empathy: $\mathrm{F}_{\mathrm{df1}, 71}=0.01, \mathrm{p}=.99$ ). Excluding the three patients with co-morbid anxiety disorder did not change the results.

\section{$M A S C$}

Separate ANOVAs were conducted to analyze the effects of spironolactone on cognitive empathy, i.e., the inferring of emotions, thoughts, and intentions in the MASC with treatment (spironolactone vs. placebo) and group (MDD vs. controls) as between subject factors.

There was no significant main or interaction effect in any of the test score (emotions: all p-values $>.23$; thoughts: all pvalues $>.50$; intentions: all p-values $>.36$ ). Mean values (SD) are presented in Table 2.

Again, there was no effect of sex (emotions: $F_{\mathrm{dfl}, 69}=0.13$, $\mathrm{p}=.71$, thoughts: $\mathrm{F}_{\mathrm{df1}, 69}=1.18, \mathrm{p}=.28$, intentions: $\mathrm{F}_{\mathrm{dfl}, 69}=$ $1.43, \mathrm{p}=.24)$. Excluding the three patients with co-morbid anxiety disorder did not change the results.

\section{Correlations between depressive symptoms and empathy}

Correlation analyses between BDI scores and MET and MASC, respectively, were performed separately for the placebo and the spironolactone condition. After placebo we found a significant positive correlation between BDI and cognitive empathy in the MET $(r=.34, p=.04)$ and a negative correlation between BDI and emotional empathy concerning positive stimuli $(\mathrm{r}=-.38, \mathrm{p}=.04)$. In the spironolactone condition a significant negative correlation between BDI and emotional empathy concerning positive stimuli occurred $(\mathrm{r}=-.45, \mathrm{p}=.009)$. 
Table 2 Cognitive empathy measured with the MASC after spironolactone administration and placebo in patients with MDD and healthy participants. There were no differences in any task score between patients and controls

\begin{tabular}{lll}
\hline MASC (mean/SD) & MDD & Healthy controls \\
\hline Placebo & $\mathrm{n}=13(7$ male) & $\mathrm{n}=22(11$ male $)$ \\
Recognition of emotions & $10.9(2.1)$ & $10.7(1.4)$ \\
Recognition of thoughts & $3.2(0.7)$ & $3.1(0.8)$ \\
Recognition of intentions & $10.4(1.5)$ & $9.6(2.1)$ \\
Spironolactone & $\mathrm{n}=14(7$ male) & $\mathrm{n}=19(9$ male $)$ \\
Recognition of emotions & $11.2(2.5)$ & $11.6(2.0)$ \\
Recognition of thoughts & $3.1(0.8)$ & $3.2(0.7)$ \\
Recognition of intentions & $10.1(2.5)$ & $10.3(2.1)$ \\
\hline
\end{tabular}

MASC Movie for the Assessment of Social Cognition, $M D D$ major depressive disorder

\section{Discussion}

We examined the effects of MR blockade via spironolactone administration on cognitive and emotional empathy in patients with MDD and in healthy individuals. Cognitive empathy was greater in depressed patients compared to healthy controls in the placebo condition. However, MR blockade with spironolactone reduced cognitive empathy of depressed patients to the level of healthy individuals. This effect was only seen in the MET, a picture-based task, but not in the MASC, a movie-based task. Concerning emotional empathy, MDD patients reported lower emotional empathy to positive but not to negative stimuli compared to controls across conditions with no effect of spironolactone.

Our results indicate that spironolactone reduced or "normalized" cognitive empathy in patients with MDD. This effect of MR blockade on cognitive empathy was not seen in healthy controls. Furthermore, we found a significant positive correlation between BDI and cognitive empathy in the MET. This leads to the question of why depressed patients exhibited higher cognitive empathy scores compared to healthy participants. Empathy is traditionally thought to be a protective factor for mental health. However, "excessive" empathy has been linked to the development of internalizing disorder and interpersonal guilt has been proposed to be a maladaptive form of cognitive empathy (Tone \& Tully, 2014). Interestingly, Wolkenstein et al. found better performance in the "Reading the Mind in the Eyes test" in depressed patients compared to controls (Wolkenstein et al., 2011). Thus, reduced as well as enhanced empathy might be maladaptive.

In contrast to the current study, we recently found that MR stimulation enhanced emotional but not cognitive empathy in healthy individuals and in patients with borderline personality disorder (Wingenfeld et al., 2014). One has to keep in mind though, that cognitive and emotional empathy are related but distinct constructs which can function independently: The ability to take another person's perspective does not predict the ability of emotional empathy (Tone \& Tully, 2014). Furthermore, different neural correlates - though interacting - underlie these two empathy constructs. Cognitive empathy is linked to the prefrontal cortex while emotional empathy is strongly associated with the limbic system, especially hippocampus, amygdale, and hypothalamus (Tone \& Tully, 2014). Thus, the role of the MR in the context of empathy and other aspects of social cognition needs to be further investigated in different populations of health and disease. In any event, however, it seems to be plausible that MR influences empathy given that the MR has been associated with the appraisal of novel situations and related response strategies as well as with stress-associated emotional reactions (de Kloet, 2013; Kruk et al., 2013; Ter Horst et al., 2012,).

What do we know about MR function and cognition in mental disorders so far? Our studies suggest: (1) improved cognitive performance in terms of memory and executive function after MR stimulation in MDD and healthy individuals (Otte et al., 2015), (2) higher emotional empathy after MR stimulation in patients with borderline personality disorder and healthy individuals (Wingenfeld et al., 2014), and (3) reduced or "normalized" cognitive empathy in patients with MDD but no changes in healthy individuals after MR blockade. Thus, it might be the case that MDD patients have an enhanced sensitivity to MR-related drug effects. However, the questions arises, why in MDD MR stimulation normalized cognition in terms of an improvement in cognitive performance, while MR blockade normalized cognitive empathy in terms of a reduction in cognitive empathy. Emotionally challenging situations and/or stress are known to facilitate a shift from higher demanding, goal-directed cognitive strategies, based for example on the prefrontal cortex and hippocampus, to more habitual action, which is more striatum-based (Schwabe \& Wolf, 2011; Vogel et al., 2016). MR blockade has to be shown to inhibit this shift (Vogel et al., 2015). Interestingly, there is compelling evidence for dysfunctional activation of the prefrontal cortex and hippocampus in MDD, possibly due to exaggerated HPA axis function (Femenia et al., 2012). Accordingly, in patients with MDD, MR blockade might strengthen or normalize frontal cortex-based cognitive evaluation strategies and, thus, normalize cognitive evaluation of emotional stimuli. In terms of MR stimulation, one might speculate that due to the high density of MR in limbic brain regions, emotional responses in terms of emotional empathy might be supported. Of note, membrane-bound MR are involved in fast cognitive effects (Khaksari et al., 2007; Schwabe et al., 2010), which fits with our results of an improvement in "cold" cognition as verbal memory and executive 
function. These effects might be mediated by MR-induced glutamate release in the hippocampus and prefrontal cortex (Joels et al., 2008). Furthermore, the release of corticotropin-releasing factor (CRF) is inhibited by $\mathrm{MR}$ stimulation (Muller et al., 2003). CRF in turn is associated with stress-associated cognitive deficits (Wang et al., 2011) and is increased in patients with major depression (Binder \& Nemeroff, 2010; Gold \& Chrousos, 2013; Holsboer \& Ising, 2010). Thus, CRF-inhibition after MR stimulation might also contribute to improved cognitive function.

Interestingly, similar to our recent study in healthy controls and patients with borderline personality disorder (Wingenfeld et al., 2014), the effects of MR manipulation were only seen in the MET and not in the MASC. Thus, the MET might be more sensitive to the effects of pharmacologic MR manipulation. This might be due to the stronger emotional impact of the stimuli presented in the MET (e.g., negative-valenced pictures like a desperate child in front of a bombed house) in comparison to the MASC, in which an "everyday situation" with relatively low emotional impact is presented. Furthermore, in the MET, relatively complex emotions have to be recognized. In the MASC social cognitive reasoning and context information might help to find the correct answers.

Patients with MDD did not differ in their test performance in the MASC compared to healthy controls. This is in line with at least one other study, which also used the MASC and did not see any deficits in depressed patients (Wilbertz et al., 2010). In contrast, another study found impaired test performance in MASC in MDD (Wolkenstein et al., 2011). However, both studies investigated relatively small samples. In contrast to our findings of reduced emotional empathy in MDD exclusively for positive stimuli, Thoma and colleagues did not find any alterations in MDD patients in the MET (Thoma et al., 2011). Overall, the literature on social cognition in MDD is rather heterogeneous (Schreiter et al., 2013). Several factors may be responsible for these inconsistencies. Task performance seems to be strongly related to the used paradigm: In one study MDD patients were found to have impaired test performance in MASC but better performance in the "Reading the Mind in the Eyes test" compared to controls (Wolkenstein et al., 2011). The latter result fits well with our finding that MDD patients had better cognitive empathy in the MET compared to controls. Interestingly, some authors emphasize that higher empathy might also have costs and will not be positive in any case or condition (Hodges \& Klein, 2001). Differences in the investigated samples might also contribute to heterogeneous results: Patients in our sample reported higher depression scores compared to the studies by Thoma et al. (2011) and Wilbertz et al. (2010). Furthermore, sex, age of first episode, and co-morbid disorder, e.g. post-traumatic stress disorder (PTSD), might influence the results (Schreiter et al., 2013). Our finding of impaired emotional empathy only for positive stimuli in depressed patients is compatible with many studies suggesting not only an attentional bias to negative emotional cues in MDD patients but also an attentional bias away from positive emotional cues (Leppanen, 2006). For example, one study reported a diminished emotional reaction to happy faces, while there was no such difference between depressed patients and controls in response to unhappy faces (Sloan et al., 2002).

A limitation of this study includes the relatively heterogeneous (sex, co-morbid disorders) but small sample size that excluded performing subgroup analyses, e.g. with regard to co-morbid mental disorders such as PTSD or with respect to sex. Both variables are known to influence empathy and HPA axis (Roepke et al., 2012; Schreiter et al., 2013; Smeets et al., 2009; Wingenfeld et al., 2010; Wingenfeld et al., 2013). The small sample size also questions the power of the study. Most of the non-significant effects are very small, i.e., $\leq .009$ and thus unlikely to be meaningful. Indeed, to find such an effect a sample size of more than 429 per group would have been needed. Furthermore, it was not possible to conduct a withinsubject design as there were no parallel versions of the used tasks. We did not assess any blood parameters, such as plasma levels of spironolactone or its metabolites, which might have been helpful for the interpretation of the results.

In summary, this is the first study demonstrating effects of MR blockade on empathy in MDD patients, showing normalization of cognitive empathy in MDD. Taken together with our recent studies suggesting memory improvement after MR stimulation, the MR could be an interesting target in the treatment of depression. However, despite a plethora of preclinical studies, human studies on MR function are still rare and the role of the MR in depression is not clear yet. Future studies should aim to replicate these results and should investigate the role of MR function in other aspects of social cognition in populations with different states of health and disease.

Acknowledgments This study was supported by a grant from the Deutsche Forschungsgemeinschaft (HI 1480/2-1) awarded to KH. It was also supported by the DFG (EXC 257 NeuroCure to CO) and NARSAD (Grant ID: 18766) awarded to KW.

Dr. Otte has received honoraria fees for lectures from Lundbeck and Servier and has received compensation as a member of the scientific advisory board from Lundbeck. Dr. Wingenfeld, Dr. Kühl, Dr. Röpke, Dr. Dziobek, and Dr. Hinkelmann report no conflicts of interest.

Compliance with ethical standards The authors assert that all procedures contributing to this work comply with the ethical standards of the relevant national and institutional committees on human experimentation and with the Helsinki Declaration of 1975, as revised in 2008. 


\section{References}

Ackenheil, M., Stotz, G., \& Dietz-Bauer, R. (1999). Mini International Neuropsychiatric Interview. German Version 5.0.0, DSM-IV. München: Psychiatrische Universitätsklinik München.

Beck, A. T., \& Steer, R. A. (1994). Beck-Depressions-Inventar: BDI. Testhandbuch. Bern: Huber.

Binder, E. B., \& Nemeroff, C. B. (2010). The CRF system, stress, depression and anxiety-insights from human genetic studies. Molecular Psychiatry, 15(6), 574-588.

Blair, R. J. (2008). Responding to the emotions of others: Dissociating forms of empathy through the study of typical and psychiatric populations. Consciousness and Cognition, 14, 698-718.

Chamberlain, S. R., \& Sahakian, B. J. (2006). The neuropsychology of mood disorders. Current Psychiatry Reports, 8(6), 458-463.

Cornelisse, S., Joels, M., \& Smeets, T. (2011). A randomized trial on mineralocorticoid receptor blockade in men: Effects on stress responses, selective attention, and memory. Neuropsychopharmacology, 36(13), 2720-2728.

de Kloet, E. R. (2010). From vasotocin to stress and cognition. European Journal of Pharmacology, 626(1), 18-26.

de Kloet, E. R. (2013). Functional profile of the binary brain corticosteroid receptor system: Mediating, multitasking, coordinating, integrating. European Journal of Pharmacology, 719(1-3), $53-62$.

de Kloet, E. R., Joels, M., \& Holsboer, F. (2005). Stress and the brain: From adaptation to disease. Nature Reviews. Neuroscience, 6(6), 463-475.

Dziobek, I., Fleck, S., Kalbe, E., Rogers, K., Hassenstab, J., Brand, M., . . Convit, A. (2006). Introducing MASC: A movie for the assessment of social cognition. Journal of Autism and Developmental Disorders, 36(5), 623-636.

Dziobek, I., Preissler, S., Grozdanovic, Z., Heuser, I., Heekeren, H. R., \& Roepke, S. (2011). Neuronal correlates of altered empathy and social cognition in borderline personality disorder. NeuroImage, 57(2), 539-548.

Dziobek, I., Rogers, K., Fleck, S., Bahnemann, M., Heekeren, H. R., Wolf, O. T., \& Convit, A. (2008). Dissociation of cognitive and emotional empathy in adults with Asperger syndrome using the Multifaceted Empathy Test (MET). Journal of Autism and Developmental Disorders, 38(3), 464-473.

Femenia, T., Gomez-Galan, M., Lindskog, M., \& Magara, S. (2012). Dysfunctional hippocampal activity affects emotion and cognition in mood disorders. Brain Research, 1476, 58-70.

Gold, P. W., \& Chrousos, G. P. (2013). Melancholic and atypical subtypes of depression represent distinct pathophysiological entities: CRH, neural circuits, and the diathesis for anxiety and depression. Molecular Psychiatry, 18(6), 632-634.

Harris, A. P., Holmes, M. C., de Kloet, E. R., Chapman, K. E., \& Seckl, J. R. (2012). Mineralocorticoid and glucocorticoid receptor balance in control of HPA axis and behaviour. Psychoneuroendocrinology, 38(5), 648-658.

Hinkelmann, K., Moritz, S., Botzenhardt, J., Riedesel, K., Wiedemann, K., Kellner, M., \& Otte, C. (2009). Cognitive impairment in major depression: Association with salivary cortisol. Biological Psychiatry, 66(9), 879-885.

Hodges, S. D., \& Klein, K. J. K. (2001). Regulating the costs of empathy: The price of beeing human. Journal of SocioEconomics, 30, 437-452.

Holsboer, F. (2000). The corticosteroid receptor hypothesis of depression. Neuropsychopharmacology, 23(5), 477-501.

Holsboer, F., \& Ising, M. (2010). Stress hormone regulation: Biological role and translation into therapy. Annual Review of Psychology, 61(81-109), C101-C111.
Joels, M., Karst, H., DeRijk, R., \& de Kloet, E. R. (2008). The coming out of the brain mineralocorticoid receptor. Trends in Neurosciences, $31(1), 1-7$.

Khaksari, M., Rashidy-Pour, A., \& Vafaei, A. A. (2007). Central mineralocorticoid receptors are indispensable for corticosterone-induced impairment of memory retrieval in rats. Neuroscience, 149(4), 729738.

Kruk, M. R., Haller, J., Meelis, W., \& de Kloet, E. R. (2013). Mineralocorticoid receptor blockade during a rat's first violent encounter inhibits its subsequent propensity for violence. Behavioral Neuroscience, 127(4), 505-514.

Leppanen, J. M. (2006). Emotional information processing in mood disorders: A review of behavioral and neuroimaging findings. Current Opinion in Psychiatry, 19(1), 34-39.

Lupien, S. J., \& Lepage, M. (2001). Stress, memory, and the hippocampus: Can't live with it, can't live without it. Behavioural Brain Research, 127(1-2), 137-158.

Muller, M. B., Zimmermann, S., Sillaber, I., Hagemeyer, T. P., Deussing, J. M., Timpl, P., . . . Wurst, W. (2003). Limbic corticotropinreleasing hormone receptor 1 mediates anxiety-related behavior and hormonal adaptation to stress. Nature Neuroscience, 6(10), 1100-1107.

Otte, C., Moritz, S., Yassouridis, A., Koop, M., Madrischewski, A. M., Wiedemann, K., \& Kellner, M. (2007). Blockade of the mineralocorticoid receptor in healthy men: Effects on experimentally induced panic symptoms, stress hormones, and cognition. Neuropsychopharmacology, 32(1), 232-238.

Otte, C., Wingenfeld, K., Kuehl, L. K., Kaczmarczyk, M., Richter, S., Quante, A., ... Hinkelmann, K. (2015). Mineralocorticoid receptor stimulation improves cognitive function and decreases cortisol secretion in depressed patients and healthy individuals. Neuropsychopharmacology, 40(2), 386-393.

Parker, K. J., Schatzberg, A. F., \& Lyons, D. M. (2003). Neuroendocrine aspects of hypercortisolism in major depression. Hormones and Behavior, 43(1), 60-66.

Porter, R. J., Bourke, C., \& Gallagher, P. (2007). Neuropsychological impairment in major depression: Its nature, origin and clinical significance. The Australian and New Zealand Journal of Psychiatry, 41(2), 115-128.

Preissler, S., Dziobek, I., Ritter, K., Heekeren, H. R., \& Roepke, S. (2010). Social cognition in borderline personality disorder: Evidence for disturbed recognition of the emotions, thoughts, and intentions of others. Frontiers in Behavioral Neuroscience, 4, 182.

Preller, K. H., Hulka, L. M., Vonmoos, M., Jenni, D., Baumgartner, M. R., Seifritz, E., . . Q Quednow, B. B. (2014). Impaired emotional empathy and related social network deficits in cocaine users. Addiction Biology, 19(3), 452-466.

Rimmele, U., Besedovsky, L., Lange, T., \& Born, J. (2013). Blocking mineralocorticoid receptors impairs, blocking glucocorticoid receptors enhances memory retrieval in humans. Neuropsychopharmacology, 38(5), 884-894.

Ritter, K., Dziobek, I., Preissler, S., Ruter, A., Vater, A., Fydrich, T., . . . Roepke, S. (2011). Lack of empathy in patients with narcissistic personality disorder. Psychiatry Research, 187(1-2), 241-247.

Roepke, S., Vater, A., Preissler, S., Heekeren, H. R., \& Dziobek, I. (2012). Social cognition in borderline personality disorder. Frontiers in Neuroscience, 6, 195.

Roozendaal, B., Hernandez, A., Cabrera, S. M., Hagewoud, R., Malvaez, M., Stefanko, D. P., . . Wood, M. A. (2010). Membrane-associated glucocorticoid activity is necessary for modulation of long-term memory via chromatin modification. The Journal of Neuroscience, 30(14), 5037-5046.

Schlosser, N., Wolf, O. T., Fernando, S. C., Riedesel, K., Otte, C., Muhtz, C., . . . Wingenfeld, K. (2010). Effects of acute cortisol administration on autobiographical memory in patients with major depression and healthy controls. Psychoneuroendocrinology, 35(2), 316-320. 
Schlosser, N., Wolf, O. T., Fernando, S. C., Terfehr, K., Otte, C., Spitzer, C., . . . Wingenfeld, K. (2013). Effects of acute cortisol administration on response inhibition in patients with major depression and healthy controls. Psychiatry Research, 209(3), 439-446.

Schlosser, N., Wolf, O. T., \& Wingenfeld, K. (2011). Cognitive correlates of HPA axis alterations and their relevance for therapeutic interventions in major depressive disorder. Expert Review of Endocrinology and Metabolism, 6(1), 109-126.

Schreiter, S., Pijnenborg, G. H., \& Aan Het Rot, M. (2013). Empathy in adults with clinical or subclinical depressive symptoms. Journal of Affective Disorders, 150(1), 1-16.

Schwabe, L., Schachinger, H., de Kloet, E. R., \& Oitzl, M. S. (2010). Corticosteroids operate as a switch between memory systems. Journal of Cognitive Neuroscience, 22(7), 1362-1372.

Schwabe, L., \& Wolf, O. T. (2011). Stress-induced modulation of instrumental behavior: From goal-directed to habitual control of action. Behavioural Brain Research, 219(2), 321-328.

Sheehan, D. V., Lecrubier, Y., Sheehan, K. H., Amorim, P., Janavs, J., Weiller, E., ... Dunbar, G. C. (1998). The Mini-International Neuropsychiatric Interview (M.I.N.I.): the development and validation of a structured diagnostic psychiatric interview for DSM-IV and ICD-10. The Journal of Clinical Psychiatry, 59(Suppl 20), 22-33; quiz 34-57.

Sloan, D. M., Bradley, M. M., Dimoulas, E., \& Lang, P. J. (2002). Looking at facial expressions: Dysphoria and facial EMG. Biological Psychology, 60(2-3), 79-90.

Smeets, T., Dziobek, I., \& Wolf, O. T. (2009). Social cognition under stress: Differential effects of stress-induced cortisol elevations in healthy young men and women. Hormones and Behavior, 55(4), 507-513.

Ter Horst, J. P., Carobrez, A. P., van der Mark, M. H., de Kloet, E. R., \& Oitzl, M. S. (2012). Sex differences in fear memory and extinction of mice with forebrain-specific disruption of the mineralocorticoid receptor. The European Journal of Neuroscience, 36(8), 3096-3102.

Terfehr, K., Wolf, O. T., Schlosser, N., Fernando, S. C., Otte, C., Muhtz, C., ... Wingenfeld, K. (2011a). Effects of acute hydrocortisone administration on declarative memory in patients with major depressive disorder: A placebo-controlled, double-blind crossover study. The Journal of Clinical Psychiatry, 72(12), 1644-1650.

Terfehr, K., Wolf, O. T., Schlosser, N., Fernando, S. C., Otte, C., Muhtz, C., . . . Wingenfeld, K. (2011b). Hydrocortisone impairs working memory in healthy humans, but not in patients with major depressive disorder. Psychopharmacology (Berl), 215(1), 71-79.

Thoma, P., Zalewski, I., von Reventlow, H. G., Norra, C., Juckel, G., \& Daum, I. (2011). Cognitive and affective empathy in depression linked to executive control. Psychiatry Research, 189(3), 373-378.

Tomova, L., von Dawans, B., Heinrichs, M., Silani, G., \& Lamm, C. (2014). Is stress affecting our ability to tune into others? Evidence for gender differences in the effects of stress on self-other distinction. Psychoneuroendocrinology, 43, 95-104.

Tone, E. B., \& Tully, E. C. (2014). Empathy as a risky strength: A multilevel examination of empathy and risk for internalizing disorders. Development and Psychopathology, 26(4 Pt 2), 1547-1565.
Vogel, S., Fernandez, G., Joels, M., \& Schwabe, L. (2016). Cognitive adaptation under stress: A case for the mineralocorticoid receptor. Trends in Cognitive Sciences, 20(3), 192-203.

Vogel, S., Klumpers, F., Kroes, M. C., Oplaat, K. T., Krugers, H. J., Oitzl, M. S., . . . Fernandez, G. (2015). A Stress-Induced Shift from Trace to Delay Conditioning Depends on the Mineralocorticoid Receptor. Biological Psychiatry, 78(12), 830-839.

von Dawans, B., Fischbacher, U., Kirschbaum, C., Fehr, E., \& Heinrichs, M. (2012). The social dimension of stress reactivity: Acute stress increases prosocial behavior in humans. Psychological Science, 23(6), 651-660.

Wang, X. D., Rammes, G., Kraev, I., Wolf, M., Liebl, C., Scharf, S. H., . . Schmidt, M. V. (2011). Forebrain CRF(1) modulates early-life stress-programmed cognitive deficits. The Journal of Neuroscience: The Official Journal of the Society for Neuroscience, 31(38), 13625-13634.

Webster, M. J., Knable, M. B., O'Grady, J., Orthmann, J., \& Weickert, C. S. (2002). Regional specificity of brain glucocorticoid receptor mRNA alterations in subjects with schizophrenia and mood disorders. Molecular Psychiatry, 7(9), 985-994. 924.

Wilbertz, G., Brakemeier, E. L., Zobel, I., Harter, M., \& Schramm, E. (2010). Exploring preoperational features in chronic depression. Journal of Affective Disorders, 124(3), 262-269.

Williams, J. B. W. (1988). A structured interview guide for the Hamilton depression rating scale. Archives of General Psychiatry, 45, 742-747.

Wingenfeld, K., Driessen, M., Terfehr, K., Schlosser, N., Fernando, S. C., Otte, C., . . . Wolf, O. T. (2013). Effects of cortisol on memory in women with borderline personality disorder: Role of co-morbid post-traumatic stress disorder and major depression. Psychological Medicine, 43(3), 495-505.

Wingenfeld, K., Kuehl, L. K., Janke, K., Hinkelmann, K., Dziobek, I., Fleischer, J., . . Roepke, S. (2014). Enhanced emotional empathy after mineralocorticoid receptor stimulation in women with borderline personality disorder and healthy women. Neuropsychopharmacology, 39(8), 1799-1804.

Wingenfeld, K., Spitzer, C., Rullkotter, N., \& Löwe, B. (2010). Borderline personality disorder: Hypothalamus pituitary adrenal axis and findings from neuroimaging studies. Psychoneuroendocrinology, 35(1), 154-170.

Wolf, O. T., Schulte, J. M., Drimalla, H., Hamacher-Dang, T. C., Knoch, D., \& Dziobek, I. (2015). Enhanced emotional empathy after psychosocial stress in young healthy men. Stress, 18(6), 631-637.

Wolkenstein, L., Schonenberg, M., Schirm, E., \& Hautzinger, M. (2011). I can see what you feel, but I can't deal with it: Impaired theory of mind in depression. Journal of Affective Disorders, 132(1-2), 104-111.

Zaki, J., \& Ochsner, K. (2012). The neuroscience of empathy: Progress, pitfalls and promise. Nature Neuroscience, 15(5), 675-680. 